\title{
Níveis de cloro da ração sobre parâmetros sanguíneos e ósseos de codornas italianas destinadas à produção de carne
}

[Chlorine levels of diet on blood parameters and bone in meat production of Italian quail]

\author{
E.R. Freitas ${ }^{1}$, R.P.S. Xavier ${ }^{2}$, G.A. Jerônimo do Nascimento ${ }^{1 *}$, A.M. Rodrigues ${ }^{1}$, \\ M.G.R. Queiroz ${ }^{1}$, D.L. Raquel ${ }^{2}$, R.C. Lima ${ }^{2}$ \\ ${ }^{1}$ Universidade Federal do Ceará - UFC - Fortaleza, CE \\ ${ }^{2}$ Aluna de pós-graduação - Universidade Federal do Ceará - UFC - Fortaleza, CE
}

\section{RESUMO}

Avaliaram-se diferentes níveis de cloro da ração sobre os parâmetros sanguíneos e o desenvolvimento ósseo de codornas destinadas à produção de carne. Foram utilizadas 384 codornas italianas de um a 49 dias de idade, distribuídas em delineamento inteiramente ao acaso, com seis tratamentos e oito repetições de oito aves. Os tratamentos consistiram de rações isonutrientes, exceto para o nível de cloro, que foram: 0,$07 ; 0,12 ; 0,17 ; 0,22 ; 0,27$ e $0,32 \%$. Aos 49 dias, foram selecionadas duas aves por parcela para as coletas do sangue e das tíbias. Foram avaliados o pH e as concentrações de $\mathrm{Na}, \mathrm{Cl}$ e $\mathrm{K}$ no sangue, bem como o comprimento, o diâmetro, o peso, o teor de matéria seca e cinzas, o índice de Seedor e a resistência à quebra e deformidade da tíbia. Os níveis de cloro da ração não influenciaram significativamente o $\mathrm{pH}$, as concentrações de $\mathrm{Na}, \mathrm{Cl}, \mathrm{K}$ no sangue, assim como o comprimento, o diâmetro, o peso, o índice de Seedor, o teor de matéria seca e cinzas e a resistência à quebra e deformidade da tíbia. Conforme os resultados, o nível de cloro pode variar de 0,07 até $0,32 \%$, sem alterar o pH e as concentrações de $\mathrm{Na}, \mathrm{Cl}$ e $\mathrm{K}$ no sangue e sem prejuízos na qualidade óssea.

Palavras-chave: desenvolvimento ósseo, equilíbrio ácido-base, índice de Seedor

\begin{abstract}
We evaluated different levels of chlorine in the feed on the blood parameters and bone development of quail for meat production. 384 Italian quails were used at 1-49 days of age, distributed in a completely randomized design with six treatments and eight replicates of eight birds. The treatments consisted of isonutrient diets, except for the level of chlorine, which were 0.07, 0.12, 0.17, 0.22, 0.27 and 0.32\%. After 49 days, two birds were selected per plot for the collection of blood and tibia. The $\mathrm{pH}$ and the concentration of $\mathrm{Na}, \mathrm{K}$ and $\mathrm{Cl}$ in the blood and the length, diameter, weight, dry matter and ash, Seedor index, breaking strength and deformity of the tibia were evaluated. The chlorine levels in the diet did not significantly influence the $\mathrm{pH}$, the concentrations of $\mathrm{Na}, \mathrm{Cl}, \mathrm{K}$ blood, length, diameter, weight, Seedor index, dry matter and ash, crack resistance and deformity of the tibia. As the results, the level of chloride can range from 0.07 to $0.32 \%$, without changing the $\mathrm{pH}$ and the concentrations of $\mathrm{Na}, \mathrm{K}$ and $\mathrm{Cl}$ in the blood and without a loss in bone quality.
\end{abstract}

Keywords: bone development, acid-base balance, Seedor index

\section{INTRODUÇÃO}

O cloro $(\mathrm{Cl})$ está incluído no grupo de minerais essenciais das rações para as aves, uma vez que exerce funções importantes no meio extracelular.
Ele é fornecido na ração das aves com a finalidade de manter a composição iônica dos fluidos corporais e por sua relação com o balanço ácido-base do animal (Cohen et al., 1972).

Recebido em 20 de abril de 2012

Aceito em 14 de maio de 2013

*Autor para correspondência (correspondência author)

E-mail: germanoaugusto@ufc.br 
O cloro, assim como o sódio e o potássio, é importante não somente por regular o equilíbrio ácido-base, mas também por influenciar o apetite, o desenvolvimento ósseo, o crescimento, a resposta ao estresse térmico e o metabolismo de certos nutrientes, como aminoácidos, minerais e vitaminas (Rodrigues et al., 2008).

O desenvolvimento adequado do sistema ósseo das aves depende do atendimento das exigências nutricionais em minerais. Entre estes, está o cloro, que, como o sódio e o potássio, tem grande importância no estabelecimento e na manutenção da pressão osmótica e do equilíbrio ácido-base dos animais e, por isso, pode influenciar na deposição mineral nos ossos.

A ração deficiente ou com excesso de cloro induz mudanças na concentração desse mineral nos tecidos e fluidos corporais; em tais circunstâncias, lesões bioquímicas devem ocorrer, funções fisiológicas serão afetadas adversamente e desordens estruturais devem surgir, variando com o grau e a duração da deficiência dietética ou toxidez, a idade, o sexo e a espécie animal (Underwood e Suttle, 1999). Segundo os pesquisadores, para prevenção dessas mudanças, o animal deve ser alimentado com dietas palatáveis e atóxicas, com níveis de cloro e de outros nutrientes em quantidades e proporções exigidas e em formas disponíveis para o organismo animal.

De acordo com Kusakawa e Faria (1998), algumas pesquisas demonstram que o excesso de cloro na ração pode levar à acidose metabólica, que, por sua vez, pode comprometer a qualidade óssea dos frangos de corte, visto que o desequilíbrio no balanço eletrolítico causado pelo excesso desse mineral pode comprometer o metabolismo da vitamina $\mathrm{D}$, reduzindo a capacidade renal em transformar o 25-hidroxicolicalciferol em 1,25-di-hidroxicalciferol.

Os relatos de problemas de pernas na criação de codornas não são comuns. Isso pode ter contribuído para que sejam poucos os estudos dos efeitos dos minerais sobre o crescimento dos ossos dessas aves.

Nesse contexto, vários pesquisadores (Vieites et al., 2004; Gonzalez e Mendonça, 2006; Araújo et al., 2011) têm relatado que os íons $\mathrm{Na}^{+}, \mathrm{K}^{+} \mathrm{e} \mathrm{Cl}^{-}$ participam da formulação das rações para as aves não só para atender os requerimentos de cada mineral, mas também para promover um balanço ótimo de eletrólitos e, assim, atender as necessidades de crescimento e evitar o aumento de problemas de pernas.

Segundo Vieites et al. (2004) e Araújo et al. (2011), os resultados das pesquisas têm demonstrado que o desequilíbrio de cátions e de ânions na ração pode ter influência na incidência de problemas de pernas em frangos, havendo interação entre sódio, potássio e cloro no desenvolvimento da discondroplasia na tíbia, sendo que o elevado teor de cloro na ração tende a aumentar a incidência desse problema, sobretudo quando os níveis de sódio e de potássio são baixos.

A coturnicultura de corte vem sendo um crescente objeto de interesse no Brasil e, consequentemente, das pesquisas para gerar conhecimento em nutrição, que viabilizem a produção eficiente. Diante do exposto, neste estudo objetivou-se avaliar o efeito do nível de cloro da ração sobre os parâmetros sanguíneos e o desenvolvimento ósseo de codornas destinadas à produção de carne.

\section{MATERIAL E MÉTODOS}

O experimento foi realizado nas instalações do setor de avicultura do Departamento de Zootecnia do Centro de Ciências Agrárias da Universidade Federal do Ceará (UFC), localizado no município de Fortaleza, estado do Ceará.

Foram utilizadas 384 codornas italianas de um dia de idade e de ambos os sexos, com peso médio inicial de $8 \pm 0,6$ gramas. As aves foram distribuídas em um delineamento inteiramente ao acaso, com seis tratamentos $(0,07,0,12,0,17$, $0,22,0,27,0,32 \%$ de cloro), oito repetições e oito aves por unidade experimental.

Inicialmente, as aves foram pesadas e distribuídas em seis boxes $(1,0 \mathrm{~m} \times 1,5 \mathrm{~m})$, sendo cada box específico para cada tratamento, com 90 codornas italianas de ambos os sexos por box, totalizando 540 codornas, que serviram para posterior seleção das 384 codornas utilizadas no estudo. Todos os boxes eram providos de aquecimento com lâmpadas incandescentes de 
100 watts e piso forrado com maravalha. As rações foram fornecidas em comedouros do tipo bandeja, e a água em bebedouros de pássaros adaptados.

No nono dia de idade, as aves foram novamente pesadas e redistribuídas, de acordo com peso médio de cada unidade experimental, em gaiolas de arame galvanizado $(52 \mathrm{~cm} \times 26 \mathrm{~cm} \times 20 \mathrm{~cm})$ contendo comedouros do tipo calha e bebedouros de pássaros adaptados. Nessa idade, as aves foram vacinadas contra a doença de Newcastle, por via ocular. Durante o período de criação, tiveram iluminação de 24 horas por dia (natural e artificial), com a iluminação noturna feita por lâmpadas fluorescentes de 40 watts. As rações e a água foram fornecidas à vontade, e os comedouros e bebedouros abastecidos duas vezes ao dia, às oito e às 16 horas.

As rações (Tab. 1) foram formuladas segundo as recomendações nutricionais em energia metabolizável, proteína bruta, aminoácidos, cálcio e fósforo constantes no NRC (National....1994). Os dados de composição dos alimentos foram baseados em Rostagno et al. (2005), e as análises realizadas no laboratório de Nutrição Animal do Departamento de Zootecnia da Universidade Federal do Ceará.

Tabela 1. Composição percentual e nutricional das dietas experimentais

\begin{tabular}{|c|c|c|c|c|c|c|}
\hline \multirow{2}{*}{ Ingrediente } & \multicolumn{6}{|c|}{ Nível de cloro $(\%)$} \\
\hline & 0,07 & 0,12 & 0,17 & 0,22 & 0,27 & 0,32 \\
\hline Milho & 53,90 & 53,97 & 54,06 & 54,00 & 53,8 & 53,68 \\
\hline Farelo de soja & 42,10 & 42,09 & 42,07 & 42,08 & 42,12 & 42,14 \\
\hline Óleo de soja & 1,11 & 1,08 & 1,05 & 1,07 & 1,14 & 1,18 \\
\hline Calcário & 1,22 & 1,22 & 1,22 & 1,22 & 1,22 & 1,22 \\
\hline Fosfato bicálcico & 0,91 & 0,91 & 0,91 & 0,91 & 0,91 & 0,91 \\
\hline Dl-Metionina & 0,01 & 0,01 & 0,01 & 0,01 & 0,01 & 0,01 \\
\hline Suplemento vitamínico e mineral ${ }^{1}$ & 0,40 & 0,40 & 0,40 & 0,40 & 0,40 & 0,40 \\
\hline Cloreto de amônia & 0,00 & 0,00 & 0,00 & 0,05 & 0,13 & 0,19 \\
\hline Bicarbonato de sódio & 0,29 & 0,17 & 0,05 & 0,00 & 0,00 & 0,00 \\
\hline Sal comum & 0,06 & 0,15 & 0,23 & 0,26 & 0,27 & 0,27 \\
\hline Total & 100,0 & 100,0 & 100,0 & 100,0 & 100,0 & 100,0 \\
\hline \multicolumn{7}{|l|}{ Composição nutricional calculada } \\
\hline Energia metabolizável (kcal/kg) & 2.900 & 2.900 & 2.900 & 2.900 & 2.900 & 2.900 \\
\hline Proteína bruta $(\%)$ & 23,80 & 23,80 & 23,80 & 23,80 & 23,80 & 23,80 \\
\hline Lisina $(\%)$ & 1,30 & 1,30 & 1,30 & 1,30 & 1,30 & 1,30 \\
\hline Metionina + cistina $(\%)$ & 0,87 & 0,87 & 0,87 & 0,87 & 0,87 & 0,87 \\
\hline Metionina (\%) & 0,50 & 0,50 & 0,50 & 0,50 & 0,50 & 0,50 \\
\hline Cálcio $(\%)$ & 0,80 & 0,80 & 0,80 & 0,80 & 0,80 & 0,80 \\
\hline Fósforo disponível (\%) & 0,30 & 0,30 & 0,30 & 0,30 & 0,30 & 0,30 \\
\hline Sódio (\%) & 0,15 & 0,15 & 0,15 & 0,15 & 0,15 & 0,15 \\
\hline Cloro $(\%)$ & 0,07 & 0,12 & 0,17 & 0,22 & 0,27 & 0,32 \\
\hline Potássio (\%) & 0,94 & 0,94 & 0,94 & 0,94 & 0,94 & 0,94 \\
\hline $\mathrm{NM}^{2}(\mathrm{mEq} / \mathrm{kg})$ & 286 & 272 & 258 & 244 & 229 & 215 \\
\hline
\end{tabular}

${ }^{1}$ Quantidade/kg do produto: ácido fólico - 138,00mg; pantotenato de cálcio - 2.750,00mg; antioxidante - 500,00mg; biotina - 13,80mg; cobalto $-25,00 \mathrm{mg}$; cobre $-2.500,00 \mathrm{mg}$; colina $-111.450,00 \mathrm{mg}$; ferro $-6.250,00 \mathrm{mg}$; iodo 260,00mg; manganês - 13.000,00mg; metionina - 300,00 g; niacina $-6.875,00 \mathrm{mg}$; piridoxina - 550,00mg; colistina - 1.750,00mg; riboflavina - 1.375,00mg; selênio - 45,00mg; tiamina - 550,00mg; vitamina A - 2.150.000,00 UI; vitamina B12 - 2.750,00mcg; vitamina D3 - 550.000 UI; vitamina E - 2.750,00 UI; vitamina K - 400,00mg; zinco $11.100,00 \mathrm{mg}$; silicatos $-20.000,00 \mathrm{mg}$.

${ }^{2}$ Número de Mongin (Mongin, 1981) $=(\% \mathrm{Na}+\times 10000 / 22,990 *)+(\% \mathrm{~K}+\times 10000 / 39,102 *)-(\% \mathrm{Cl}-\times$ $10000 / 35,453 *$ ) (*equivalente-grama do sódio, potássio e cloro). 
Os níveis de $\mathrm{Na}(0,15 \%)$ e K $(0,94 \%)$ nas rações foram mantidos constantes em todas as rações; os diferentes níveis de cloro foram obtidos variando-se a inclusão de cloreto de sódio $(\mathrm{NaCl})$ e cloreto de amônio (NH4Cl); e o bicarbonato de sódio (NaHCO3) foi utilizado para manter os mesmos níveis de sódio nas rações.

Diariamente, durante todo o período experimental, a temperatura e a umidade relativa do ar dentro do galpão foram medidas com termômetro de máxima e mínima e psicrômetro, respectivamente. Os dados foram registrados diariamente, e as leituras realizadas às oito e às 16 horas. No final do experimento, foram calculadas as médias das temperaturas máximas e mínimas e os valores de umidade relativa do ar.

Ao final do período experimental (49 dias de idade), foram selecionadas duas aves de cada parcela experimental, com peso próximo ao peso médio da parcela para a coleta de sangue e retirada das tíbias.

Os parâmetros sanguíneos avaliados foram: $\mathrm{pH}$ e concentração dos minerais $\mathrm{Na}(\mathrm{mmol} / \mathrm{L})$, $\mathrm{Cl}(\mathrm{mmol} / \mathrm{L})$ e $\mathrm{K}(\mathrm{mmol} / \mathrm{L})$, bem como os parâmetros ósseos: comprimento $(\mathrm{cm})$, diâmetro $(\mathrm{cm})$ peso $(\mathrm{mg})$ índice de Seedor $(\mathrm{mg} / \mathrm{mm})$, matéria seca e cinzas $(\%)$, deformidade $(\mathrm{mm})$ e resistência óssea $\left(\mathrm{kgf} / \mathrm{cm}^{2}\right)$.

Para a coleta de sangue, as aves de cada parcela foram retiradas da gaiola e decapitadas com o auxílio de uma tesoura. O sangue de cada ave foi coletado em copo plástico descartável, sendo aproximadamente $3 \mathrm{~mL}$ colhidos com seringa, colocados imediatamente em tubos coletores de sangue contendo gel separador e deixados para coagular por cerca de 40 minutos em temperatura ambiente. Em seguida, procedeu-se à centrifugação das amostras a 20000rpm por 15 minutos e, após a separação do soro, estas foram imediatamente congeladas em freezer $\left(-20^{\circ} \mathrm{C}\right)$ até realização das análises laboratoriais. A parte restante foi utilizada para determinação do $\mathrm{pH}$, com a imersão direta do eletrodo do peagâmetro no sangue contido no copo.

As determinações de sódio, cloretos e potássio no soro sanguíneo foram realizadas no Laboratório de Análises Clínicas e Toxicológicas (LACT) da Faculdade de Farmácia, Odontologia e Enfermagem da UFC, por técnicas fotocolorimétricas utilizando-se kits reagentes da marca Labtest (Labtest Diagnóstica, Brasil). As determinações de sódio, potássio e cloro foram realizadas em equipamento automático (Rapidchem 744) da marca Bayer, por meio do princípio de íon eletrodo seletivo.

Após a sangria, foram retiradas as coxas e as sobrecoxas de cada ave, identificadas, pesadas e congeladas para posterior desossa e análise dos parâmetros ósseos.

Para a realização da desossa, as peças foram descongeladas, pesadas, devidamente identificadas e mergulhadas em água fervente por 10 minutos. Em seguida, foram desossadas com auxílio de um bisturi, conforme metodologia descrita por Bruno (2002).

Depois da desossa, nas tíbias do lado direito, foram medidos o comprimento e o diâmetro, com auxílio de um paquímetro digital, e determinouse o peso em balança de precisão $(0,01 \mathrm{~g})$. A densidade óssea $(\mathrm{mg} / \mathrm{mm})$ foi calculada por meio do índice de Seedor, obtido dividindo-se o valor do peso (mg) pelo comprimento ( $\mathrm{mm}$ ) do osso avaliado (Seedor et al. 1991).

Após a pesagem, os ossos foram colocados em estufa de ventilação forçada a $55^{\circ} \mathrm{C}$ por $72 \mathrm{~h}$ para a determinação da matéria pré-seca. Em seguida, os ossos foram triturados em moinho tipo bola, devidamente identificados e acondicionados para posterior determinação da matéria seca (MS) e matéria mineral (MM) segundo as metodologias descritas por Silva e Queiroz (2002).

A resistência e a deformidade óssea foram determinadas no osso in natura (tíbia esquerda) com auxílio de uma prensa mecânica. Os ossos foram colocados em posição horizontal sobre um suporte de madeira, apoiados sob suas extremidades, e depois foi aplicada uma força de compressão no centro de cada osso. A quantidade máxima de força aplicada no osso antes da sua ruptura foi considerada a resistência à quebra $\left(\mathrm{kgf} / \mathrm{cm}^{2}\right)$, sendo esta mensurada por um extensômetro digital. A deformidade (mm) também foi mensurada por um extensômetro no momento da ruptura do osso.

Os dados obtidos foram submetidos à análise estatística usando-se o programa estatístico computacional "Statistical Analysis Sistem" 
(Statistical..., 2001), e as estimativas dos melhores níveis de cloro foram estabelecidas por meio do modelo de regressão.

\section{RESULTADOS E DISCUSSÃO}

Conforme a análise dos dados (Tab. 2), não houve efeito significativo dos níveis de cloro sobre o $\mathrm{pH}$ e as concentrações de $\mathrm{Na}, \mathrm{Cl}$ e $\mathrm{K}$ no sangue.

Os resultados obtidos para as codornas são semelhantes aos relatados por Mushtaq et al. (2005) para frangos de corte na fase de um a 28 dias de idade. Esses pesquisadores verificaram que o $\mathrm{pH}$ e as concentrações de $\mathrm{Na}$ e $\mathrm{K}$ do sangue não foram influenciados pelo nível de $\mathrm{Cl}$ da ração. Entretanto Mushtaq et al. (2007) constataram que o aumento de $0,30 \%$ para $0,40 \%$ de cloro na ração de frangos de corte submetidos ao estresse por calor na fase de 29 a 42 dias de idade promoveu redução do $\mathrm{pH}$ e das concentrações de $\mathrm{Na}$ e $\mathrm{K}$ do sangue.

Sabe-se que o aumento dos níveis de $\mathrm{Cl}$ na ração pode induzir a acidose metabólica nas aves, pois favorece a retenção íons H+ no sangue (Mushtaq et al., 2005 e Mushtaq et al., 2007). Entretanto, segundo os pesquisadores, os efeitos dos níveis de $\mathrm{Cl}$ da ração sobre os parâmetros sanguíneos podem variar, pois os mecanismos regulatórios do equilíbrio ácido-base são dinâmicos e dependem das relações entre o $\mathrm{Cl}$ e os demais eletrólitos corporais, bem como das condições ambientais em que as aves estão sendo criadas.

Tabela 2. pH e eletrólitos do sangue de codornas italianas destinadas à produção de carne, alimentadas com diferentes níveis de cloro

\begin{tabular}{ccccc}
\hline & \multicolumn{4}{c}{ Parâmetros $^{1}$} \\
\cline { 2 - 5 } Níveis de cloro (\%) & $\mathrm{pH}$ & $\mathrm{Na}(\mathrm{mmol} / \mathrm{L})$ & $\mathrm{Cl}(\mathrm{mmol} / \mathrm{L})$ & $\mathrm{K}(\mathrm{mmol} / \mathrm{L})$ \\
\hline 0,07 & 7,17 & 132,28 & 106,66 & 4,39 \\
0,12 & 7,26 & 133,80 & 106,56 & 4,46 \\
0,17 & 7,24 & 135,63 & 106,19 & 4,32 \\
0,22 & 7,13 & 137,71 & 105,44 & 4,39 \\
0,27 & 7,10 & 134,82 & 104,79 & 4,25 \\
0,32 & 7,24 & 139,83 & 108,61 & 4,28 \\
Média & 7,08 & 135,89 & 106,37 & 4,35 \\
CV $^{2}(\%)$ & 1,05 & 4,04 & 4,25 & 11,11 \\
Regressão & $\mathrm{NS}$ & $\mathrm{NS}$ & $\mathrm{NS}$ \\
\hline
\end{tabular}

pH=pH sanguíneo; $\mathrm{Na}=$ sódio $(\mathrm{mmol} / \mathrm{L}) ; \mathrm{Cl}=$ cloro $(\mathrm{mmol} / \mathrm{L}) ; \mathrm{K}=$ potássio $(\mathrm{mmol} / \mathrm{L}) ;{ }^{2} \mathrm{CV}=$ coeficiente de variação; ${ }^{3} \mathrm{NS}=$ efeito estatístico não significativo $(\mathrm{P}>0,05)$.

Dessa forma, pode-se inferir que as variações nos níveis de $\mathrm{Cl}$ avaliadas não foram suficientes para alterar a capacidade das codornas em manter o equilíbrio ácido-base e demonstram que não houve deficiência ou excesso.

Conforme a análise dos dados dos parâmetros ósseos avaliados (Tab. 3), os níveis de cloro da ração não influenciaram significativamente o comprimento, o diâmetro, o peso, o índice de Seedor, o teor de matéria seca e cinzas e a resistência à quebra e deformidade da tíbia.

Nos relatos da literatura (Kussakawa e Faria, 1998; Underwood e Suttle, 1999), observa-se que o aumento do nível de cloro da ração reduz o balanço eletrolítico das rações e pode contribuir para a acidose metabólica e, assim, influenciar no desenvolvimento do tecido ósseo, prejudicando a qualidade dos ossos das aves. $\mathrm{Na}$ presente pesquisa os níveis de $0,07,0,12,0,17$, $0,22,0,27,0,32 \%$ de cloro resultaram em rações com balanço eletrolítico de 286, 272, 258, 243, 229 e $215 \mathrm{mEq} / \mathrm{kg}$ de ração, respectivamente.

Embora tenha ocorrido redução do balanço eletrolítico com o aumento do cloro, esses resultados estão em desacordo daqueles apresentados pelos pesquisadores anteriormente citados, já que não ocorreu influência estatística significativa na qualidade óssea das codornas analisadas. Pode-se inferir, portanto, que a redução do balanço eletrolítico até $215 \mathrm{mEq} / \mathrm{kg}$ de ração para codornas não foi suficiente para que ocorressem os prejuízos ósseos relatados pelos pesquisadores. 
Niveis de cloro da ração...

Tabela 3. Efeito dos diferentes níveis de cloro sobre os parâmetros ósseos avaliados na tíbia de codornas italianas

\begin{tabular}{lccccccccc}
\hline & \multicolumn{10}{c}{ Níveis de cloro (\%) } \\
\cline { 2 - 7 } & 0,07 & 0,12 & 0,17 & 0,22 & 0,27 & 0,32 & Média & CV $^{2}(\%)$ & REG $^{3}$ \\
\hline COMP (cm) & 53,79 & 52,51 & 52,77 & 52,13 & 52,58 & 52,70 & 52,72 & 3,34 & NS $^{4}$ \\
DIAM (cm) & 0,318 & 0,304 & 0,301 & 0,303 & 0,301 & 0,318 & 0,307 & 9,67 & NS \\
PESO (mg) & 656,0 & 652,0 & 620,0 & 628,3 & 682,5 & 683,3 & 653,4 & 11,3 & NS \\
ISE (mg/mm) & 12,20 & 12,43 & 11,74 & 12,05 & 12,99 & 12,92 & 12,39 & 10,58 & NS \\
MS (\%) & 77,34 & 79,88 & 78,38 & 75,94 & 76,73 & 77,33 & 77,59 & 10,11 & NS \\
CZ (\%) & 46,91 & 46,84 & 46,54 & 46,42 & 46,62 & 47,76 & 46,85 & 5,05 & NS \\
DEF (mm) & 1,41 & 1,44 & 1,44 & 1,45 & 1,42 & 1,43 & 1,43 & 17,45 & NS \\
RES (kgf/cm $\left.{ }^{2}\right)$ & 1,72 & 1,71 & 1,71 & 1,73 & 1,73 & 1,73 & 1,72 & 22,27 & NS \\
\hline
\end{tabular}

${ }^{1} \mathrm{COMP}=$ comprimento $(\mathrm{cm})$; DIAM $=$ diâmetro $(\mathrm{cm})$; PESO= Peso $(\mathrm{mg})$; ISE $=$ índice de Seedor $(\mathrm{mg} / \mathrm{mm})$; $\mathrm{MS}=$ matéria seca $(\%) ; \mathrm{CZ}=$ cinzas $(\%) ; \mathrm{DEF}=$ deformidade $(\mathrm{mm}) ; \mathrm{RES}=$ resistência $\left(\mathrm{kgf} / \mathrm{cm}^{2}\right) ;{ }^{2} \mathrm{CV}=$ coeficiente de variação $(\%) ;{ }^{3} \mathrm{REG}=$ regressão; ${ }^{4} \mathrm{NS}=$ efeito estatístico não significativo $(\mathrm{P}>0,05)$.

A ausência de influência dos níveis de cloro sobre o desenvolvimento dos ossos longos também foi relatada por Murakami et al. (2001), que avaliaram a influência do cloro na ração $(0,05, \quad 0,10,0,15,0,20$ e $0,25 \%$ de $\mathrm{Cl})$ na incidência de discondroplasia tibial em frangos de corte.

Araújo et al. (2011) verificaram que a redução no balanço eletrolítico da ração de frangos de corte influenciou negativamente a mineralização e a resistência óssea, sendo a redução na resistência à quebra dos ossos associada não apenas às alterações na mineralização, mas também a mudanças na composição da matriz orgânica do osso devido a alterações nas concentrações de proteínas colagenosas e não colagenosas, que estão correlacionadas negativamente com a resistência à quebra.

Alterações nos níveis de cálcio no sangue de frangos de corte em resposta a modificações do balanço eletrolítico na ração foram relatadas por Vieites et al. (2004), em cujo trabalho os tratamentos com os menores e os maiores valores de BE apresentaram as maiores concentrações de fósforo e de cálcio no sangue, o que pode representar maior mobilização óssea desses minerais por ação hormonal, com a finalidade de disponibilizar mais eletrólitos no sangue para serem utilizados por mecanismos compensatórios de regulação no organismo das aves.

Segundo Gonzalez e Mendonça (2006), o balanço eletrolítico ótimo para reduzir os problemas locomotores em frangos de corte deve estar entre 250 e $300 \mathrm{mEq} / \mathrm{kg}$, corroborando Johnson e Karunajeewa (1985), os quais concluíram que um balanço de eletrólitos na dieta menor que $180 \mathrm{mEq} / \mathrm{kg}$ e maior que $300 \mathrm{mEq} / \mathrm{kg}$ deprimiu o peso das aves, quando avaliado aos 42 dias de idade. No entanto um ótimo balanço eletrolítico foi encontrado para rações contendo de 250 a $300 \mathrm{mEq} / \mathrm{kg}$.

\section{CONCLUSÕES}

O aumento do nível de cloro pode variar de 0,07 para $0,32 \%$ na ração de codornas de corte sem, contudo, alterar o pH e as concentrações de eletrólitos no sangue e sem apresentar prejuízos na qualidade óssea até 49 dias de idade.

\section{REFERÊNCIAS}

ARAÚJO, G.M.; VIEITES, F.M.; BARBOSA, A.A. et al. Variação aniônica da dieta sobre características ósseas de frangos de corte: resistência à quebra, composição orgânica e mineral. Arq. Bras. Med. Vet. Zootec., v.63, p.954-961, 2011.

BRUNO, L.G.D. Desenvolvimento ósseo em frangos de corte: Influência da restrição alimentar e da temperatura ambiente. 2002. 72f. Tese (Doutorado) - Universidade Estadual Paulista, Jaboticabal, SP.

COHEN, L.; HURWITZ, S.; BAR, A. Acid-base balance and sodium to chloride ratio in diets of laying hens. J. Nutr., v.102, p.1-7, 1972. 
GONZALEZ, E.; MENDONÇA, C.X. Problemas locomotores em frangos de corte. In: SIMPÓSIO BRASIL SUL DE AVICULTURA, 7., 2006, Chapecó. Anais... Chapecó: Embrapa Suínos e Aves, 2006. p.79-94.

JOHNSON, R.J.; KARUNAJEEWA, H. The effects of dietary minerals and electrolytes on the growt and physiology of the young chick. $J$. Nutr., v.115, p.1680-1690, 1985.

KUSSAKAWA, K.C.K.; FARIA, H.G. Discondroplasia tibial em frangos de corte: aspectos nutricionais. Arq. Cienc. Saúde Unipar, v.2, p.275-282, 1998.

MONGIN, P. Recent advances in dietary cationanion balance: applications in poultry. Proceed. Nutrit. Society, v.40, p.285-294, 1981.

MURAKAMI, A.E.; OVIEDO RONDON, E.O.; MARTINS, E.N. et al. Sodium and Chloride requeriments of growing broiler chikens (twentyone to forty-two days of age) fes cornsoybean diets. Poult. Sci., v.80, p.289-294, 2001.

MUSHTAQ, T.; MIRZA, M.A.; ATHAR, M. et al. Dietary sodium and chloride for twenty-nine to forty-twoday-old broiler chickens at constant electrolyte balance under subtropical summer conditions. J. Applied Poult. Res., v.16, p.161170, 2007.

MUSHTAQ, T.; SARWAR, M.; NAWAZ, H. et al. Effect and interactions of dietary sodium and chloride on broiler starter performance (hatching to twenty-eight days of age) under subtropical summer conditions. Poult. Sci., v.84, p.17161722, 2005.
NATIONAL Research Council - NRC. Nutrient requirements of poultry. 9.ed. Washington: National Academy Press, 1994. 155p.

RODRIGUES, V.P.; COSTA, F.G.P.; GOULART, C.C. et al. Exigência de cloro para codornas japonesas de 1 a 21 dias de idade. In: CONGRESSO BRASILEIRO DE ZOOTECNIA, 18.; CONGRESSO INTERNACIONAL DE ZOOTECNIA, 10., 2008, João Pessoa. Anais... João Pessoa: Universidade Federal da Paraíba, 2008. CDROM.

ROSTAGNO, H.S.; ALBINO, L.F.T.; DONZELE, J.L. et al. Tabelas Brasileiras para aves e suínos: composição de alimentos e exigências nutricionais. 2.ed. Viçosa: UFV, 2005. 186p.

SEEDOR, J.G.; QUARRACCIO, H.H; THOMPSON, D.D. The biophosphonate alendronate (MK-217) inhibits bone loss due to ovariectomy in rats. Bone and Mineral Res., v.6, p.339-346, 1991.

SILVA, D.J.; QUEIROZ, A.C. Análise de alimentos, métodos químicos e biológicos. Viçosa: Editora UFV, 2.ed., 2002. 235p.

STATISTICAL Analysis System. SAS User's Guide, 8.ed. Cary: SAS Institute, 2001.

UNDERWOOD, E.J.; SUTTLE, N.F. General introduction. In:__. Mineral nutrition of Livestock. Wageningen: CABI, 1999. p.1-16.

VIEITES, F.M.; MORAES, G.H.K.; ALBINO, L.F.T. et al. Balanço Eletrolítico e Níveis de Proteína Bruta sobre Parâmetros Sanguíneos e Ósseos de Frangos de Corte aos 21 Dias de Idade. Rev. Bras. Zootec., v.33, p.1520-1530, 2004. 